\title{
Unsteady gravity-driven slender rivulets of a power-law fluid
}

\author{
Y. M. Yatim, S. K. Wilson, B. R. Duffy* \\ Department of Mathematics and Statistics, University of Strathclyde, \\ Livingstone Tower, 26 Richmond Street, Glasgow G1 1XH, United Kingdom
}

\begin{abstract}
Unsteady gravity-driven flow of a thin slender rivulet of a non-Newtonian power-law fluid on a plane inclined at an angle $\alpha$ to the horizontal is considered. Unsteady similarity solutions are obtained for both converging sessile rivulets (when $0<\alpha<\pi / 2$ ) in the case $x<0$ with $t<0$, and diverging pendent rivulets (when $\pi / 2<\alpha<\pi$ ) in the case $x>0$ with $t>0$, where $x$ denotes a coordinate measured down the plane and $t$ denotes time. Numerical and asymptotic methods are used to show that for each value of the power-law index $N$ there are two physically realisable solutions, with cross-sectional profiles that are 'single-humped' and 'double-humped', respectively. Each solution predicts that at any time $t$ the rivulet widens or narrows according to $|x|^{(2 N+1) / 2(N+1)}$ and thickens or thins according to $|x|^{N /(N+1)}$ as it flows down the plane; moreover, at any station $x$, it widens or narrows according to $|t|^{-N / 2(N+1)}$ and thickens or thins according to $|t|^{-N /(N+1)}$. The length of a truncated rivulet of fixed volume is found to behave according to $|t|^{N /(2 N+1)}$.
\end{abstract}

Keywords: Power-law fluid, rivulet, similarity solution, unsteady flow

\section{Introduction}

Thin films of non-Newtonian fluids occur in many situations, ranging from geophysical contexts such as flows of lava and mud to industrial situations such as lubrication and coating processes (see, for example, Ferguson and Kemblowski [1], Chhabra and Richardson [2] and Tanner [3]). There is therefore a considerable literature on thin-film flows of non-Newtonian fluids, both with prescribed rigid boundaries (as is common in, for example, lubrication problems) and with unknown free surfaces (as in, for example, mud flows). In this paper we shall be concerned with similarity solutions for flows of the latter type.

\footnotetext{
${ }^{*}$ Corresponding author.

Email addresses: s.k.wilson@strath.ac.uk (S. K. Wilson), b.r.duffy@strath.ac.uk (B. R. Duffy)
} 
The simplest types of non-Newtonian fluid models are the so-called generalized Newtonian models, in which the extra stress is proportional to the rate-of-strain tensor, with the viscosity $\mu$ a prescribed function of the local shear rate $q$; of these, the simplest is the power-law fluid, in which $\mu$ is proportional to $q^{N-1}$, where $N(>0)$ is a constant (the power-law index). Power-law fluids may be shear-thinning $(N<1)$ or shear-thickening $(N>1)$, and can be useful models of non-Newtonian behaviour of many materials over restricted ranges of values of $q$; the case $N=1$ corresponds to a Newtonian fluid. Barnes et al. [4] give examples of materials that exhibit power-law behaviour, such as toothpaste and skin cream (which are shear-thinning) and anti-misting solution in jet fuel (which is shear-thickening). Myers [5] gives comparisons between predictions of several generalized Newtonian fluids (including power-law fluids) in some rectilinear flows, and Hamrock [6] and Szeri [7] describe the use of power-law fluids in modeling non-Newtonian lubricants in geometries with rigid boundaries.

There have been many studies of steady flows of thin films of power-law fluids with a free surface. For example, following the approach of Smith [8] for a rivulet of a Newtonian fluid, Wilson and Burgess [9] obtained a similarity solution for the steady gravity-driven spreading of a rivulet of a power-law fluid down an inclined plane which predicts that the rivulet widens according to $x^{(2 N+1) /(5 N+2)}$ and thins according to $x^{-N /(5 N+2)}$ (where $x$ is a longitudinal coordinate), but that the profile of the rivulet is rather insensitive to $N$. Wilson and Burgess [9] noted a formal analogy between their steady three-dimensional rivulet problem and an unsteady onedimensional nonlinear diffusion problem (the time variable $t$ being replaced by a space variable), and Perazzo and Gratton [10] used this analogy to obtain solutions to a variety of free-surface flow problems, and, in particular, to recover the similarity solution of Wilson and Burgess [9] from a known solution of the diffusion problem given by Zel'dovich and Kompaneets [11] and Pattle [12]. Wilson et al. [13] obtained similarity solutions for the steady spreading of a rivulet of a power-law fluid driven by either gravity or a constant shear stress at its free surface, for both weak and strong surface-tension effects.

There have also been studies of unsteady flows of thin films of power-law fluids with a free surface. Gorodtsov [14] obtained similarity solutions describing unsteady two-dimensional and axisymmetric gravity-driven spreading of a power-law fluid on a horizontal plane, generalizing the earlier solutions for a Newtonian fluid given by Smith [15] (for the case when the volume of fluid is constant) and Huppert [16] (for the case when the volume changes according to some 
power of time $t$ ). The solutions obtained by Gorodtsov [14] were re-discovered independently by Pascal [17] (in the two-dimensional constant-volume case), by Pascal [18] (in the axisymmetric constant-volume case), and by Gratton et al. [19]. Gorodtsov [14] also obtained a similarity solution describing spreading of a power-law fluid on a horizontal plane with vertical sidewall boundaries, and Gratton et al. [19] obtained a variety of waiting-time and travelling-wave solutions for thin-film flows of a power-law fluid on a horizontal plane. Perazzo and Gratton [20] obtained a similarity solution describing unsteady two-dimensional gravity-driven flow of a power-law fluid down an inclined plane which predicts that the height of the free surface varies

according to $(x / t)^{N /(N+1)}$, generalizing the result given by Huppert [21] for the Newtonian case $N=1$. Perazzo and Gratton [20,22] also obtained families of travelling-wave solutions for thinfilm flows of a power-law fluid on an inclined plane. Pascal [23] and Pascal and D'Alessio [24] investigated the stability of flow of a power-law fluid down a porous inclined plane, and down an inclined plane subject to a prescribed shear stress at its free surface, respectively.

There have also been studies of both steady and unsteady thin-film flows of power-law fluids in which surface-tension effects are significant. For example, Witelski [25] derived similarity solutions relevant to spreading and contracting flows, and Betelú and Fontelos [26, 27] obtained similarity solutions describing spreading in two-dimensional and axisymmetric geometries, respectively.

In this paper, we use the general approach of our previous work on rivulets of a Newtonian fluid (Yatim et al. [28]) to obtain similarity solutions describing unsteady gravity-driven draining of a rivulet of a non-Newtonian power-law fluid down an inclined plane.

\section{Problem Formulation}

Consider the unsteady flow of a thin slender rivulet of a non-Newtonian power-law fluid with constant density $\rho$ and variable viscosity $\mu=\mu_{0} q^{N-1}$, where $\mu_{0}(>0)$ is a constant, $q$ is the local shear rate and $N(>0)$ is the power-law index, down a planar substrate inclined at an angle $\alpha(0<\alpha<\pi)$ to the horizontal subject to gravitational acceleration $g$ when surfacetension effects are negligible. When $0<\alpha<\pi / 2$ the fluid is on the upper side of the inclined plane (a sessile rivulet), and when $\pi / 2<\alpha<\pi$ it is on the underside of the inclined plane (a pendent rivulet).

Cartesian coordinates $(x, y, z)$ with the $x$ axis down the line of greatest slope and the $z$ axis 
normal to the substrate are adopted, with the substrate at $z=0$. We denote the free surface profile of the rivulet by $z=h(x, y, t)$, where $t$ denotes time, and we restrict our attention to flows that are symmetric about $y=0$ (i.e. to solutions for which $h$ is even in $y$ ) with (unknown) semi-width $a=a(x, t)$, so that $h=0$ at the contact lines $y= \pm a$. The geometry of the problem is sketched in Fig. .1.

With the usual lubrication approximation the velocity $(u, v, w)$ and pressure $p$ of the fluid satisfy the governing equations

$$
\begin{gathered}
u_{x}+v_{y}+w_{z}=0 \\
\left(\mu u_{z}\right)_{z}+\rho g \sin \alpha=0 \\
\left(\mu v_{z}\right)_{z}-p_{y}=0 \\
-p_{z}-\rho g \cos \alpha=0
\end{gathered}
$$

Since the rivulet is taken to be thin and slender, and since we will be considering only problems in which $u_{z}$ is always non-negative, the shear rate is given by $q=u_{z}$ approximately, and therefore the viscosity $\mu$ in (2) and (3) is given by $\mu=\mu_{0} u_{z}^{N-1}$. Equations (1)-(4) are to be integrated subject to the boundary conditions of no slip and no penetration on the substrate:

$$
u=v=w=0 \quad \text { on } \quad z=0,
$$

and balances of normal and tangential stress on the free surface:

$$
p=p_{a}, \quad \mu u_{z}=\mu v_{z}=0 \quad \text { on } \quad z=h,
$$

where $p_{a}$ denotes atmospheric pressure, together with the kinematic condition on the free surface:

$$
h_{t}+\bar{u}_{x}+\bar{v}_{y}=0 \quad \text { on } \quad z=h
$$

and the zero-mass-flux conditions at the contact lines:

$$
\bar{v}= \pm a_{x} \bar{u} \quad \text { at } \quad y= \pm a
$$

where the local fluxes $\bar{u}=\bar{u}(x, y, t)$ and $\bar{v}=\bar{v}(x, y, t)$ are defined by

$$
\bar{u}=\int_{0}^{h} u \mathrm{~d} z, \quad \bar{v}=\int_{0}^{h} v \mathrm{~d} z .
$$

Integration of (2)-(4) subject to boundary conditions (5) and (6) yields

$$
p=p_{a}+\rho g \cos \alpha(h-z),
$$




$$
\begin{gathered}
u=\frac{N}{N+1}\left(\frac{\rho g \sin \alpha}{\mu_{0}}\right)^{\frac{1}{N}}\left[h^{\frac{N+1}{N}}-(h-z)^{\frac{N+1}{N}}\right], \\
v=-\frac{N}{N+1} p_{y}\left(\frac{(\rho g \sin \alpha)^{1-N}}{\mu_{0}}\right)^{\frac{1}{N}}\left[h^{\frac{N+1}{N}}-(h-z)^{\frac{N+1}{N}}\right],
\end{gathered}
$$

and substitution of (11) and (12) into (9) gives

$$
\bar{u}=\frac{N}{2 N+1}\left(\frac{\rho g \sin \alpha}{\mu_{0}}\right)^{\frac{1}{N}} h^{\frac{2 N+1}{N}}, \quad \bar{v}=-\frac{N p_{y}}{2 N+1}\left(\frac{(\rho g \sin \alpha)^{1-N}}{\mu_{0}}\right)^{\frac{1}{N}} h^{\frac{2 N+1}{N}} .
$$

The kinematic condition (7) then yields the governing partial differential equation for $h$, namely

$$
\frac{2 N+1}{N} \mu_{0}\left(\frac{\rho g \sin \alpha}{\mu_{0}}\right)^{\frac{N-1}{N}} h_{t}=\rho g \cos \alpha\left[h^{\frac{2 N+1}{N}} h_{y}\right]_{y}-\rho g \sin \alpha\left[h^{\frac{2 N+1}{N}}\right]_{x} .
$$

Once this is solved for $h$, the complete solution for $p, u$ and $v$ is given by (10)-(12). Note that in the special case $N=1$, equation (14) reduces to the familiar equation describing the unsteady gravity-driven flow of a thin slender rivulet of Newtonian fluid. From (13) we have $\bar{u}=0$ at $y= \pm a$, so that the zero-mass-flux condition (8) reduces to $\bar{v}=0$ at $y= \pm a$; thus we have the contact-line conditions

$$
h=0 \quad \text { at } \quad y= \pm a, \quad h^{\frac{2 N+1}{N}} h_{y} \rightarrow 0 \quad \text { as } \quad y \rightarrow \pm a .
$$

We shall be concerned with unsteady similarity solutions of (14) of the form

$$
h=h_{0}\left|\frac{x}{t}\right|^{\frac{N}{N+1}} H(\eta), \quad y=y_{0}\left(\frac{|x|^{2 N+1}}{|t|^{N}}\right)^{\frac{1}{2(N+1)}} \eta
$$

where $H(\geq 0)$ and $\eta$ are defined to be dimensionless, and $h_{0}$ and $y_{0}$ are positive constants, which, without loss of generality, we may write as

$$
h_{0}=\left(\frac{\mu_{0}}{\rho g \sin \alpha}\right)^{\frac{1}{N+1}}, \quad y_{0}=\left(\frac{N+1}{2 N+1}\right)^{\frac{1}{2}}\left(\frac{\mu_{0}|\cos \alpha|^{N+1}}{\rho g \sin ^{N+2} \alpha}\right)^{\frac{1}{2(N+1)}} .
$$

Hence (14) reduces to the ordinary differential equation

$$
S_{t}\left[\frac{1}{2} \eta H^{\prime}-H\right]=S_{g}\left[H^{\frac{2 N+1}{N}} H^{\prime}\right]^{\prime}+S_{x}\left[\frac{1}{2} \eta\left(H^{\frac{2 N+1}{N}}\right)^{\prime}-H^{\frac{2 N+1}{N}}\right]
$$

for $H$, where a dash denotes differentiation with respect to $\eta$, and we have introduced the notation $S_{t}=\operatorname{sgn}(t)= \pm 1, S_{g}=\operatorname{sgn}(\cos \alpha)= \pm 1$ and $S_{x}=\operatorname{sgn}(x)= \pm 1$ (so that, in particular, $S_{g}=+1$ for a sessile rivulet and $S_{g}=-1$ for a pendent rivulet). 
For a symmetric rivulet, appropriate boundary and symmetry conditions are

$$
H=H_{0}, \quad H^{\prime}=0 \quad \text { at } \quad \eta=0,
$$

where $H_{0}(>0)$ is a free parameter at this stage; the (unknown) position of the contact line is denoted by $\eta=\eta_{0}$, so that with (15) we have

$$
H=0 \quad \text { at } \quad \eta=\eta_{0}, \quad H^{\frac{2 N+1}{N}} H^{\prime} \rightarrow 0 \quad \text { as } \quad \eta \rightarrow \eta_{0} .
$$

The middle height of the rivulet, $h_{\mathrm{m}}=h(x, 0, t)$, and the semi-width of the rivulet vary with $x$ and $t$ according to

$$
h_{\mathrm{m}}=h_{0}\left|\frac{x}{t}\right|^{\frac{N}{N+1}} H_{0}, \quad a=y_{0}\left(\frac{|x|^{2 N+1}}{|t|^{N}}\right)^{\frac{1}{2(N+1)}} \eta_{0}
$$

predicting that at any time $t$ the rivulet widens or narrows according to $|x|^{(2 N+1) / 2(N+1)}$ and thickens or thins according to $|x|^{N /(N+1)}$ as it flows down the plane; moreover, at any station $x$, it widens or narrows according to $|t|^{-N / 2(N+1)}$ and thickens or thins according to $|t|^{-N /(N+1)}$.

The volume flux of fluid across any section $x=$ constant, denoted by $Q$, is given by

$$
Q=2 \int_{0}^{a} \bar{u} \mathrm{~d} y=\frac{N(N+1)^{\frac{1}{2}}}{(2 N+1)^{\frac{3}{2}}}\left(\frac{\mu_{0}^{3}|\cos \alpha|^{N+1}|x|^{3(2 N+1)}}{\rho^{3} g^{3} \sin ^{N+4} \alpha|t|^{5 N+2}}\right)^{\frac{1}{2(N+1)}} I
$$

and the cross-sectional area of the rivulet at any station $x$, denoted by $A$, is given by

$$
A=2 \int_{0}^{a} h \mathrm{~d} y=\left(\frac{N+1}{2 N+1}\right)^{\frac{1}{2}}\left(\frac{\mu_{0}^{3}|\cos \alpha|^{N+1}|x|^{4 N+1}}{\rho^{3} g^{3} \sin ^{N+4} \alpha|t|^{3 N}}\right)^{\frac{1}{2(N+1)}} J
$$

where the constants $I$ and $J$ are defined by

$$
I=2 \int_{0}^{\eta_{0}} H^{\frac{2 N+1}{N}} \mathrm{~d} \eta, \quad J=2 \int_{0}^{\eta_{0}} H \mathrm{~d} \eta
$$

Conditions for the rivulet to be thin and slender are that the length scales in the $x, y$ and $z$ directions (namely $|x|, a$ and $h_{\mathrm{m}}$, respectively) satisfy $h_{\mathrm{m}} \ll a \ll|x|$, which in turn requires that

$$
\frac{|x||t|^{N} \rho g|\cos \alpha|^{N+1}}{\mu_{0} \sin ^{N} \alpha} \gg 1, \quad \frac{|x||t|^{N} \rho g \sin ^{N+2} \alpha}{\mu_{0}|\cos \alpha|^{N+1}} \gg 1,
$$

showing that $|x||t|^{N}$ must be sufficiently large (and that $\alpha$ cannot be close to $0, \pi / 2$ or $\pi$ ). In addition, conditions for the neglect of the down-slope pressure gradient $p_{x}$ in $(2)$ and of surface-tension effects in $(6)_{1}$ are

$$
\left|h_{x}\right| \ll 1, \quad \frac{\rho g x^{2}}{\sigma} \gg 1,
$$


respectively. In principle, all these restrictions are achieved at sufficiently large length scales in the $x$ direction.

For simplicity in plotting results, we now re-scale according to

$$
\begin{gathered}
x=X x^{*}, \quad y=y_{0}\left(\frac{X^{2 N+1}}{T^{N}}\right)^{\frac{1}{2(N+1)}} y^{*}, \quad z=h_{0}\left(\frac{X}{T}\right)^{\frac{N}{N+1}} z^{*}, \quad t=T t^{*}, \\
h=h_{0}\left(\frac{X}{T}\right)^{\frac{N}{N+1}} h^{*}, \quad h_{\mathrm{m}}=h_{0}\left(\frac{X}{T}\right)^{\frac{N}{N+1}} h_{\mathrm{m}}^{*}, \quad a=y_{0}\left(\frac{X^{2 N+1}}{T^{N}}\right)^{\frac{1}{2(N+1)}} a^{*}, \\
Q=\frac{N(N+1)^{\frac{1}{2}}}{(2 N+1)^{\frac{3}{2}}}\left(\frac{\mu_{0}^{3}|\cos \alpha|^{N+1} X^{3(2 N+1)}}{\rho^{3} g^{3} \sin ^{N+4} \alpha T^{5 N+2}}\right)^{\frac{1}{2(N+1)}} Q^{*}, \\
A=\left(\frac{N+1}{2 N+1}\right)^{\frac{1}{2}}\left(\frac{\mu_{0}^{3}|\cos \alpha|^{N+1} X^{4 N+1}}{\rho^{3} g^{3} \sin ^{N+4} \alpha T^{3 N}}\right)^{\frac{1}{2(N+1)}} A^{*},
\end{gathered}
$$

where $X(>0)$ and $T(>0)$ are length and time scales, respectively, which we may choose arbitrarily. Then, with superscript stars dropped immediately for clarity, the solution (16) takes the slightly simpler form

$$
h=\left|\frac{x}{t}\right|^{\frac{N}{N+1}} H(\eta), \quad y=\left(\frac{|x|^{2 N+1}}{|t|^{N}}\right)^{\frac{1}{2(N+1)}} \eta,
$$

with $H$ satisfying (18)-(20); from (21) the middle height $h_{\mathrm{m}}$ and semi-width $a$ are given by

$$
h_{\mathrm{m}}=\left|\frac{x}{t}\right|^{\frac{N}{N+1}} H_{0}, \quad a=\left(\frac{|x|^{2 N+1}}{|t|^{N}}\right)^{\frac{1}{2(N+1)}} \eta_{0},
$$

and from (22) and (23) the flux $Q$ and cross-sectional area $A$ become

$$
Q=\left(\frac{|x|^{3(2 N+1)}}{|t|^{5 N+2}}\right)^{\frac{1}{2(N+1)}} I, \quad A=\left(\frac{|x|^{4 N+1}}{|t|^{3 N}}\right)^{\frac{1}{2(N+1)}} J .
$$

\section{Results}

A closed-form solution of the ordinary differential equation (18) is not available, and so it must, in general, be solved numerically for $H$ subject to the boundary conditions (19) and (20), where $H_{0}$ and $\eta_{0}$ are parameters to be determined.

As far as the differential equation (18) is concerned, any choice of a set of values of $S_{t}, S_{g}$ and $S_{x}$ leads to the same mathematical problem as the set $-S_{t},-S_{g}$ and $-S_{x}$ (though the two sets of values lead to different physical interpretations of the solutions, as we shall show). Therefore there are four distinct cases to consider; however, we shall show below that only the case $S_{t}=-S_{g}=S_{x}$ can lead to physically realisable solutions. 
Near $\eta=0$ we find straightforwardly that $H$ satisfies

$$
H=H_{0}+\frac{S_{x} H_{0}^{\frac{N+1}{N}}-S_{t}}{2 S_{g} H_{0}^{\frac{N+1}{N}}} \eta^{2}+O\left(\eta^{4}\right)
$$

as $\eta \rightarrow 0$, and near the contact line $\eta=\eta_{0}$ we find that $H$ satisfies either

$$
H \sim\left[-\frac{(2 N+1) S_{t} \eta_{0}}{2 N S_{g}}\left(\eta_{0}-\eta\right)\right]^{\frac{N}{2 N+1}}
$$

valid only when $S_{t}=-S_{g}$, or

$$
H \sim C\left(\eta_{0}-\eta\right)^{\frac{N}{3 N+1}}-\frac{(3 N+1) S_{t} \eta_{0}}{2(4 N+1) C^{\frac{N+1}{N}} S_{g}}\left(\eta_{0}-\eta\right)^{\frac{2 N}{3 N+1}}
$$

as $\eta \rightarrow \eta_{0}$, where $C$ is a positive constant. ${ }^{1}$ However, the zero-mass-flux condition in (20) requires that $C=0$, and so cases where $S_{t}=S_{g}$, in which (33) is the only possible behaviour near the contact line, are immediately eliminated. Determining the physical solutions from the condition $C=0$ will be discussed shortly.

In cases where $S_{t}=-S_{g}$, at any stationary points of the free surface (where $H^{\prime}=0$ ) equation (18) gives

$$
H^{\prime \prime}=H^{-\frac{N+1}{N}}-S_{x} S_{t}
$$

provided that $H \neq 0$. Since the rivulet has two contact lines, at $\eta= \pm \eta_{0}$, the function $H(\geq 0)$ must have at least one maximum in $|\eta| \leq \eta_{0}$. In the case where $S_{t}=-S_{g}=-S_{x}$, equation (34) shows that any stationary point of $H$ is a minimum, so there can be no solution for $H$ with a maximum, and therefore this case is eliminated. In the case where $S_{t}=-S_{g}=S_{x}$, equation (34) shows that any stationary point for which $H<1(H>1)$ is a minimum (maximum); later, we will show from the numerically determined profiles $H$ that there are in fact only two types of solution in this case, one with a minimum at $\eta=0$ and two symmetrically placed maxima when $H_{0}<1$ ('double-humped' profiles), and one with a single maximum at $\eta=0$ when $H_{0}>1$ ('single-humped' profiles).

With all other cases eliminated, the only case that could lead to physically realisable solutions is the one where $S_{t}=-S_{g}=S_{x}$, corresponding to a converging sessile rivulet in $x<0$ for $t<0$, or a diverging pendent rivulet in $x>0$ for $t>0$. In this case, equation (18) becomes

$$
\frac{N}{3 N+1}\left(H^{\frac{3 N+1}{N}}\right)^{\prime \prime}=\frac{1}{2} \eta\left(H^{\frac{2 N+1}{N}}-H\right)^{\prime}-\left(H^{\frac{2 N+1}{N}}-H\right)
$$

\footnotetext{
${ }^{1}$ Note that both (32) and (33) have $H^{\prime} \rightarrow \infty$ as $\eta \rightarrow \eta_{0}$, showing that the lubrication approximation always fails near the contact lines.
} 
which was solved numerically by means of a shooting method subject to (19) to find $\eta_{0}$ for a given value of $H_{0}(>0)$. As a check, some of the computations were also performed by means of a finite-difference method, with Newton iteration to solve the resulting nonlinear algebraic equations; the solutions obtained in this way were found to be in good agreement with those obtained by the shooting method. Figure .2 shows $\eta_{0}$ as a function of $H_{0}$ for several values of $N$. For each value of $N$ there is a solution for every $H_{0}$ except in a narrow 'window' $H_{01}<H_{0}<H_{02}$, where $H_{01}=H_{01}(N)<1$ and $H_{02}=H_{02}(N)>1$, in which there is no solution. This window is larger for larger values of $N$, with $H_{01}$ and $H_{02}$ taking correspondingly smaller and larger values, respectively. For example, for $N=1 / 20$, we found that $H_{01} \simeq 0.9999$ and $H_{02} \simeq 1.0178$, whereas for $N=20$, we found that $H_{01} \simeq 0.9993$ and $H_{02} \simeq 1.1409 ;$ furthermore, in the Newtonian case $(N=1), H_{01} \simeq 0.9995$ and $H_{02} \simeq 1.1059$, in agreement with the values given by Yatim et al. [28]. (Here and subsequently, quantities obtained numerically are given to four decimal places.)

In order to choose the physically realisable solutions from this family of solutions, we impose the condition (20), or equivalently the condition $C=0$. A simple way to determine $C$ accurately is by integrating equation (18) from $\eta=0$ to $\eta=\eta_{0}$ and using (19) and (20) to obtain

$$
\frac{3(3 N+1)}{2 N} \int_{0}^{\eta_{0}}\left(H^{\frac{2 N+1}{N}}-H\right) \mathrm{d} \eta= \begin{cases}0 & \text { for }(32) \\ C^{\frac{3 N+1}{N}} & \text { for }(33)\end{cases}
$$

so that the coefficient $C$ in $(33)$ is given by

$$
C=\left[\frac{3(3 N+1)}{2 N} \int_{0}^{\eta_{0}}\left(H^{\frac{2 N+1}{N}}-H\right) \mathrm{d} \eta\right]^{\frac{N}{3 N+1}}=\left[\frac{3(3 N+1)}{4 N}(I-J)\right]^{\frac{N}{3 N+1}}
$$

which we use to find $C$ from the numerical solution for $H$ obtained with a given value of $H_{0}$. Also these values of $C$ were checked against those obtained from the behaviour of the numerical solution near $\eta=\eta_{0}$, according to (33). Figure .3 shows a plot of $C$ calculated from (37) as a function of $H_{0}$ for the values of $N$ used in Fig. .2. From this plot, we see that for each value of $N$ there are precisely two values of $H_{0}$ for which $C=0$, namely $H_{0}=H_{01}$ and $H_{0}=H_{02}$. Thus we arrive at our main result: there are precisely two physically realisable solutions of the type sought for an unsteady rivulet, these solutions corresponding to the two values $H_{0}=H_{01}$ (with associated $\eta_{0}=\eta_{01}$ ) and $H_{0}=H_{02}$ (with associated $\eta_{0}=\eta_{02}$ ).

Equation (37) shows that $I$ and $J$ satisfy $I=J$ for both $H_{0}=H_{01}$ and $H_{0}=H_{02}$, and Figure .4 shows plots of $I(=J)$ as a function of $N$. 
Figures .5 and .6 show numerically calculated profiles $H=H(\eta)$ of the two physically realisable solutions with $H_{0}=H_{01}$ and $H_{0}=H_{02}$, respectively, for a range of values of $N$. These two sets of solutions have different types of profiles, namely a (barely discernible) doublehumped profile for $H_{0}=H_{01}$ and a single-humped profile for $H_{0}=H_{02}$, consistent with the earlier discussion of stationary points.

Figure .7 shows a plot of $H_{01}$ and $H_{02}$ as functions of $N$; the inset shows an enlargement of the $H_{01}$ curve, as the details are hard to distinguish at this scale. Figure .8 shows a plot of $\eta_{01}$ and $\eta_{02}$ as functions of $N$; we see that $\eta_{01}>\eta_{02}$ for all $N$.

The behaviour of the solution for $H$ in the case $H_{0}=H_{02}$ in the limit of a strongly shearthinning fluid, $N \rightarrow 0^{+}$, is discussed in the Appendix, in which it is shown that

$$
H \sim 1+N \log \left[\frac{3}{2}\left(1-\frac{\eta^{2}}{\eta_{02}^{2}}\right)\right], \quad H_{02} \sim 1+N \log \frac{3}{2}, \quad \eta_{02} \sim \sqrt{6 N}, \quad I=J \sim 2 \sqrt{6 N} .
$$

The asymptotic forms of $H_{02}$ and $\eta_{02}$ in the limit $N \rightarrow 0^{+}$given in (38) are included in Figs .7 and .8 as dashed curves, and similarly the asymptotic forms for $I(=J)$ are included in Fig. .4 as dashed curves. Numerical calculations indicate that the behaviour of the solution for $H$ in the case $H_{0}=H_{01}$ in the limit $N \rightarrow 0^{+}$is somewhat similar to (38) but with multiplicative factors and terms in $\log N$ which the authors have not been able to determine with certainty.

In the limit of a strongly shear-thickening fluid, $N \rightarrow \infty$, the solutions for both $H_{0}=H_{01}$ and $H_{0}=H_{02}$ may be expressed as regular expansions in powers of $1 / N$ about the solutions for $1 / N=0$, and so we write

$H(\eta)=\bar{H}_{0}+\frac{1}{N} \bar{H}_{1}+O\left(\frac{1}{N^{2}}\right), \quad H_{0 k}=\bar{H}_{00}+\frac{1}{N} \bar{H}_{01}+O\left(\frac{1}{N^{2}}\right), \quad \eta_{0 k}=\bar{\eta}_{00}+\frac{1}{N} \bar{\eta}_{01}+O\left(\frac{1}{N^{2}}\right)$

for $k=1$ and 2. Then equation (35) gives

$$
\frac{1}{2} \eta \bar{H}_{0}^{\prime}-\bar{H}_{0}=-\left(\bar{H}_{0}^{2} \bar{H}_{0}^{\prime}\right)^{\prime}+\frac{1}{2} \eta\left(\bar{H}_{0}^{2}\right)^{\prime}-\bar{H}_{0}^{2}
$$

at leading order in $1 / N$, and

$$
\left(\bar{H}_{0}^{2} \bar{H}_{1}\right)^{\prime \prime}+\frac{1}{2} \eta\left[\left(1-2 \bar{H}_{0}\right) \bar{H}_{1}\right]^{\prime}-\left(1-2 \bar{H}_{0}\right) \bar{H}_{1}=\left(\frac{1}{2} \eta \bar{H}_{0}^{\prime}-\bar{H}_{0}\right) \log \bar{H}_{0}-\bar{H}_{0} \bar{H}_{0}^{\prime}\left(\bar{H}_{0}^{\prime}-\frac{1}{2} \eta\right)
$$

at first order. Equations (40) and (41) were solved numerically subject to the boundary conditions

$$
\bar{H}_{0}(0)=\bar{H}_{00}, \quad \bar{H}_{0}^{\prime}(0)=0, \quad \bar{H}_{1}(0)=\bar{H}_{01}, \quad \bar{H}_{1}^{\prime}(0)=0,
$$


where the constants $\bar{H}_{00}$ and $\bar{H}_{01}$ are chosen so that conditions obtained from (37) with $C=0$, namely

$$
\int_{0}^{\bar{\eta}_{00}}\left(\bar{H}_{0}^{2}-\bar{H}_{0}\right) \mathrm{d} \eta=0
$$

and

$$
\int_{0}^{\bar{\eta}_{00}}\left[\bar{H}_{1}\left(1-2 \bar{H}_{0}\right)-\bar{H}_{0}^{2} \log \bar{H}_{0}\right] \mathrm{d} \eta=0
$$

are satisfied. For $H_{0}=H_{01}$, we find that $\bar{H}_{00} \simeq 0.9993, \bar{\eta}_{00} \simeq 9.2811$ and $\bar{H}_{01} \simeq 0.0002592$; for $H_{0}=H_{02}$, we find that $\bar{H}_{00} \simeq 1.1434, \bar{\eta}_{00} \simeq 2.5190$ and $\bar{H}_{01} \simeq-0.05084$. Figure .9 shows comparisons between $\bar{H}_{1}$ and $N\left(H-\bar{H}_{0}\right)$ for $N=5$ and $N=20$, respectively, for $H_{0}=H_{01}$, and Fig. 10 shows the corresponding results for $H_{0}=H_{02}$; the asymptotic solutions are in good agreement with the exact numerical solutions for both $H_{0}=H_{01}$ and $H_{0}=H_{02}$. The corresponding asymptotic forms of $H_{01}, H_{02}, \eta_{01}$ and $\eta_{02}$ in the limit $N \rightarrow \infty$ are included in Figs .7 and .8 as dashed curves. Also using these asymptotic solutions we find that

$$
I \sim 2 \int_{0}^{\bar{\eta}_{00}}\left[\bar{H}_{0}^{2}+\frac{1}{N}\left(\bar{H}_{0}^{2} \log \bar{H}_{0}+2 \bar{H}_{0} \bar{H}_{1}\right)\right] \mathrm{d} \eta, \quad J \sim 2 \int_{0}^{\bar{\eta}_{00}}\left(\bar{H}_{0}+\frac{1}{N} \bar{H}_{1}\right) \mathrm{d} \eta
$$

in the limit $N \rightarrow \infty$, so that

$$
I=J \sim a+\frac{1}{N} b
$$

where $a \simeq 18.4903$ and $b \simeq-9.1214$ for $H_{0}=H_{01}$, and $a \simeq 4.7084$ and $b \simeq-2.3697$ for $H_{0}=H_{02}$; these asymptotic solutions for $I(=J)$ in the limit $N \rightarrow \infty$ are included in Fig. .4 as dashed curves.

\section{Discussion}

We have obtained unsteady similarity solutions for gravity-driven flow of a thin slender rivulet of a power-law fluid down an inclined plane. We found that there are physically realisable solutions only in the case $S_{t}=-S_{g}=S_{x}$, and then there are two distinct solutions for each value of the power-law index $N$, namely one for which $H_{0}=H_{01}$, with a double-humped crosssectional profile, and one for which $H_{0}=H_{02}$, with a single-humped cross-sectional profile.

Physically, the case $S_{t}=-S_{g}=S_{x}=1$ corresponds to pendent rivulets in $x>0$, with $t>0$, whereas the case $S_{t}=-S_{g}=S_{x}=-1$ corresponds to sessile rivulets in $x<0$, with $t<0$. However, results of a numerical study of the stability of corresponding similarity solutions for a Newtonian fluid (Yatim et al. [28]) suggest that the sessile case is stable but that the pendent case is unstable. Presumably the same is true in the non-Newtonian case, 
that is, only the sessile case $S_{t}=-S_{g}=S_{x}=-1$ is likely to be stable. Figure 11 shows three-dimensional plots of the free surface $z=h$ in the cases $H_{0}=H_{01}$ and $H_{0}=H_{02}$ for $N=5$, at times $t=-100,-10$ and -1 , for the sessile case $S_{t}=-S_{g}=S_{x}=-1$; the insets show the cross-sectional profiles. Each solution predicts that at time $t$ the rivulet (in $x<0$ ) narrows according to $(-x)^{(2 N+1) / 2(N+1)}$ and thins according to $(-x)^{N /(N+1)}$ as it flows down the plane, the 'nose' of the rivulet remaining stationary at $O$ at all time. At any station $x$, the rivulet widens according to $(-t)^{N / 2(N+1)}$ and thickens according to $(-t)^{N /(N+1)}$, for $t<0$; at $t=0$, both solutions exhibit a finite-time singularity, becoming infinite everywhere at that instant.

So far we have interpreted each of our solutions as representing an infinitely long rivulet, involving an infinite volume of fluid. In the spirit of Huppert's [21] analysis, we may alternatively interpret each solution as representing a rivulet of (prescribed) finite volume, by truncating it at some finite length $L=L(t)$, so that it occupies $-L \leq x \leq 0$. The volume of fluid in such a rivulet, denoted by $V$, is given by

$$
V=\int_{-L}^{0} \int_{-a}^{a} h \mathrm{~d} y \mathrm{~d} x=\frac{2}{3}\left(\frac{N+1}{2 N+1}\right)^{\frac{3}{2}}\left(\frac{\mu_{0}^{3}|\cos \alpha|^{N+1} L^{3(2 N+1)}}{\rho^{3} g^{3} \sin ^{N+4} \alpha|t|^{3 N}}\right)^{\frac{1}{2(N+1)}} J
$$

thus the length $L$ of the rivulet at time $t$ is given by

$$
L=\left[\left(\frac{2 N+1}{N+1}\right)^{N+1}\left(\frac{9 V^{2} \sin \alpha}{4 J^{2}|\cos \alpha|}\right)^{\frac{N+1}{3}} \frac{\rho g \sin \alpha}{\mu_{0}}\right]^{\frac{1}{2 N+1}}|t|^{\frac{N}{2 N+1}}
$$

decreasing like $|t|^{N /(2 N+1)}$.

Lastly, we also used the approach described above to investigate whether there are corresponding similarity solutions for the rather different physical context of unsteady gravity-driven flow of an infinitely wide thin film of a power-law fluid around a symmetric slender dry patch $|y|<a(x, t)$ on an inclined plane (so that the fluid occupies $|y| \geq a(x, t)$, and its free surface $z=h$ again satisfies (15)). In that case the governing equation (14) again holds, and if we seek a similarity solution of the form $(16)$ then $H(\eta)$ again satisfies the ordinary differential equation (18). We now look for a solution $H$ satisfying $H \rightarrow H_{\infty}$ as $\eta \rightarrow \infty$, where $H_{\infty}$ is a positive constant; then equation (18) immediately gives $S_{t} H_{\infty}=S_{x} H_{\infty}^{\frac{2 N+1}{N}}$, which requires both that $S_{t}=S_{x}$ and that $H_{\infty}=1$, so that the appropriate far-field conditions for (18) are $H \rightarrow 1$ and $H^{\prime} \rightarrow 0$ as $\eta \rightarrow \infty$. Moreover, $H$ must again satisfy the contact-line conditions (20), where $\eta_{0}$ again denotes the position of the contact line. 
Consideration of the far-field behaviour of $H$ shows that only the case $S_{t}=S_{g}=S_{x}$ could possibly lead to physical solutions. From equation (18) with $S_{t}=S_{g}=S_{x}$, at any stationary

point of the free surface we have $H^{\prime \prime}=1-H^{-\frac{N+1}{N}}$, showing that any stationary point with $H<1(H>1)$ would be a maximum (minimum); we conclude that $H$ must therefore increase monotonically from $H=0$ at $\eta=\eta_{0}$ to $H \rightarrow 1$ as $\eta \rightarrow \infty$, with $0 \leq H<1$.

Near the contact line $\eta=\eta_{0}$, behaviour (32) still holds for a dry patch but now with $S_{t}=S_{g}$, whereas behaviour (33) is replaced by

$$
H \sim C\left(\eta-\eta_{0}\right)^{\frac{N}{3 N+1}}-\frac{(3 N+1) \eta_{0}}{2(4 N+1) C^{\frac{N+1}{N}}}\left(\eta-\eta_{0}\right)^{\frac{2 N}{3 N+1}},
$$

where $C$ is a positive constant; however, as in the earlier analysis of rivulet flow, the contact-line conditions then require that $C=0$, so that (49) is eliminated, and only (32) need be considered. Then integrating equation (18) from $\eta=\eta_{0}$ to $\eta=\infty$ gives

$$
\int_{\eta_{0}}^{\infty}\left(H-H^{\frac{2 N+1}{N}}\right) \mathrm{d} \eta=0
$$

which is impossible for monotonic $H$ satisfying $0 \leq H<1$. We therefore conclude there are no dry-patch solutions of the type sought.

\section{Acknowledgment}

The first author (YMY) wishes to thank the Ministry of Higher Education (MOHE), Malaysia and University of Science, Malaysia for financial support via an Academic Staff Training Fellowship.

\section{References}

[1] J. Ferguson, Z. Kemblowski, Applied Fluid Rheology, Elsevier, London, 1991.

[2] R. P. Chhabra, J. F. Richardson, Non-Newtonian Flow in the Process Industries, Butterworth-Heinemann, Oxford, 1999.

[3] R. I. Tanner, Engineering Rheology (2nd ed.), Oxford University Press, 2000.

[4] H. A. Barnes, J. F. Hutton, K. Walters, An Introduction to Rheology, Elsevier Science Publishers B. V., Amsterdam, 1989. 
[5] T. G. Myers, Application of non-Newtonian models to thin film flow, Phys. Rev. E $\mathbf{7 2}$ (2005) 066302-1-066302-11.

[6] B. J. Hamrock, Fundamentals of Fluid Film Lubrication, McGraw-Hill, New York, 1994.

[7] A. Z. Szeri, Fluid Film Lubrication: Theory and Design, Cambridge University Press, Cambridge, 1998.

[8] P. C. Smith, A similarity solution for slow viscous flow down an inclined plane, J. Fluid Mech. 58 (1973) 275-288.

[9] S. D. R. Wilson, S. L. Burgess, The steady, spreading flow of a rivulet of mud, J. NonNewtonian Fluid Mech. 79 (1998) 77-85.

[10] C. A. Perazzo, J. Gratton, Exact solutions for two-dimensional steady flows of a power-law liquid on an incline, Phys. Fluids 17 (2005) 013102-1-013102-8.

[11] Ya. B. Zel'dovich, A. S. Kompaneets, On the theory of heat propagation for temperaturedependent thermal conductivity, Collection Commemorating the 70th Anniversary of A. F. Joffe, Izv. Akad. Nauk SSSR (1950) 61-71.

[12] R. E. Pattle, Diffusion from an instantaneous point source with a concentration-dependent coefficient, Q. Jl Mech. Appl. Math. 12 (1959) 407-409.

[13] S. K. Wilson, B. R. Duffy, R. Hunt, A slender rivulet of a power-law fluid driven by either gravity or a constant shear stress at the free surface, Q. Jl Mech. Appl. Math. 55 (2002) 385-408.

[14] V. A. Gorodtsov, Spreading of a film of nonlinearly viscous liquid over a horizontal smooth solid surface, J. Engrg Phys. 57 (1990) 879-884.

[15] S. H. Smith, On initial value problems for the flow in a thin sheet of viscous liquid, J. Appl. Math. Phys. 20 (1969) 556-560.

[16] H. E. Huppert, The propagation of two-dimensional and axisymmetric viscous gravity currents over a rigid horizontal surface, J. Fluid Mech. 121 (1982) 43-58.

[17] H. Pascal, Gravity flow of a non-Newtonian fluid sheet on an inclined plane, Int. J. Engng Sci. 29 (1991) 1307-1313. 
[18] H. Pascal, Similarity solutions to some unsteady flows of non-Newtonian fluids of power law behaviour, Int. J. Non-linear Mech. 27 (1992) 759-771.

[19] J. Gratton, F. Minotti, S. M. Mahajan, Theory of creeping gravity currents of a nonNewtonian liquid, Phys. Rev. E 60 (1999) 6960-6967.

[20] C. A. Perazzo, J. Gratton, Thin film of non-Newtonian fluid on an incline, Phys. Rev. E 67 (2003) 016307-1-016307-6.

[21] H. E. Huppert, Flow and instability of a viscous current down a slope, Nature 300 (1982) $427-429$.

[22] C. A. Perazzo, J. Gratton, Steady and traveling flows of a power-law liquid over an incline, J. Non-Newtonian Fluid Mech. 118 (2004) 57-64.

[23] J. P. Pascal, Instability of power-law fluid flow down a porous incline, J. Non-Newtonian Fluid Mech. 133 (2006) 109-120.

[24] J. P. Pascal, S. J. D. D'Alessio, Instability of power-law fluid flows down an incline subjected to wind stress, Appl. Math. Modelling 31, 1229-1248 (2007).

[25] T. P. Witelski, Similarity solutions of the lubrication equation, Appl. Math. Lett. 10 (1997) $107-113$.

[26] S. I. Betelú, M. A. Fontelos, Capillarity driven spreading of power-law fluids, Appl. Math. Lett. 16 (2003) 1315-1320.

[27] S. I. Betelú, M. A. Fontelos, Capillarity driven spreading of circular drops of shear-thinning fluid, Mathl Comput. Modelling 40 (2004) 729-734.

[28] Y. M. Yatim, B. R. Duffy, S. K. Wilson, R. Hunt, Similarity solutions for unsteady gravitydriven slender rivulets, submitted to Q. Jl Mech. Appl. Math.

\section{Appendix}

In this Appendix we derive equation (38) giving the behaviour of the solution for $H$ in the case $H_{0}=H_{02}$ in the limit of a strongly shear-thinning fluid, $N \rightarrow 0^{+}$. 
For the case in which $H_{0}=H_{02}$ and $N$ is small, our numerical solutions suggest that $\eta_{0} \sim \sqrt{N}$ and that $H=1+O(N)$ and $H^{\frac{2 N+1}{N}}=O(1)$ except very close to the contact line $\eta=\eta_{0}$. Therefore in the limit $N \rightarrow 0^{+}$we re-scale $H$ and $\eta$ according to

$$
H=1+N \hat{h}(\hat{\eta}), \quad \eta=\sqrt{N} \hat{\eta}, \quad \eta_{02}=\sqrt{N} \hat{\eta}_{02} .
$$

With the result $\lim _{N \rightarrow 0}(1+N \hat{h})^{1 / N}=e^{\hat{h}}=O(1)$, equation (35) then yields

$$
\left(e^{\hat{h}}\right)^{\prime \prime}-\frac{1}{2} \hat{\eta}\left(e^{\hat{h}}\right)^{\prime}+e^{\hat{h}}=1
$$

at leading order in $N$, whose solution subject to the boundary condition $\hat{h}^{\prime}(0)=0$ is

$$
\hat{h}=\log \left(e^{\hat{h}_{0}}+\frac{1-e^{\hat{h}_{0}}}{2} \hat{\eta}^{2}\right),
$$

where $\hat{h}_{0}=\hat{h}(0)=\left(H_{02}-1\right) / N(>0)$ is the (unknown) value of $\hat{h}$ at $\hat{\eta}=0$. The solution (.3) is valid provided that $e^{\hat{h}_{0}}+\frac{1}{2}\left(1-e^{\hat{h}_{0}}\right) \hat{\eta}^{2}>0$, which requires $\hat{\eta}<\hat{\eta}_{02}$, where

$$
\hat{\eta}_{02}=\left(\frac{2 e^{\hat{h}_{0}}}{e^{\hat{h}_{0}}-1}\right)^{\frac{1}{2}},
$$

and we note that $\hat{h}=0$ at $\hat{\eta}=\sqrt{2}$, and that $\hat{\eta}_{02}>\sqrt{2}$.

To determine $\hat{h}_{0}$ we use equation (37) with $C=0$ (that is, $I=J$ ), leading to $\hat{h}_{0}=\log (3 / 2)$. Then equations (.3) and (.4) become

$$
\hat{h}=\log \left(\frac{6-\hat{\eta}^{2}}{4}\right), \quad \hat{\eta}_{02}=\sqrt{6}
$$

and hence to first order we have

$$
H \sim 1+N \log \left[\frac{3}{2}\left(1-\frac{\eta^{2}}{\eta_{02}^{2}}\right)\right], \quad \eta_{02} \sim \sqrt{6 N}, \quad H_{02} \sim 1+N \log \frac{3}{2}
$$

in the limit $N \rightarrow 0^{+}$. Lastly the integrals $I$ and $J$ in (24) satisfy

$$
I \sim 2 \int_{0}^{\sqrt{N} \hat{\eta}_{02}} \frac{6 N-\eta^{2}}{4 N} \mathrm{~d} \eta=2 \sqrt{6 N}, \quad J \sim 2 \sqrt{N} \hat{\eta}_{02}=2 \sqrt{6 N}
$$

in the limit $N \rightarrow 0^{+}$; there is a boundary layer in the solution for $H$ near $\eta=\eta_{0}$, but it does not contribute to these integrals to this order.

A sketch of the asymptotic solution for $H$ given in (.6) is shown in Fig. .12, and Fig. .13 shows a comparison between profiles $H$ computed numerically (represented by full curves) and the asymptotic solution (.6) in the limit $N \rightarrow 0^{+}$(represented by dashed curves) in the cases $N=1 / 2$ and $N=1 / 20$; the asymptotic solution is in good agreement with the exact numerical solution away from the contact line. 


\section{Figure captions}

Fig. .1. Sketch of the geometry of the problem.

Fig. .2. Plot of $\eta_{0}$ as a function of $H_{0}$ for $N=1 / 20,1 / 10,1 / 5,1 / 2,1,2,5,10$ and 20 .

Fig. .3. Plot of $C$ in (37) as a function of $H_{0}$ for $N=1 / 20,1 / 10,1 / 5,1 / 2,1,2,5,10$ and 20.

Fig. .4. Plot of $I(=J)$ given by (24) as a function of $N$ for $H_{0}=H_{01}$ and $H_{0}=H_{02}$, together with the asymptotic solutions (38) in the limit $N \rightarrow 0^{+}$and (46) in the limit $N \rightarrow \infty$, represented by dashed curves.

Fig. .5. Numerically calculated profiles $H=H(\eta)$ for the case $H_{0}=H_{01}$, for $N=1 / 20,1 / 10$, $1 / 5,1 / 2,1,2,5,10$ and 20 .

Fig. .6. As in Fig. .5, but for the case $H_{0}=H_{02}$.

Fig. .7. Plots of $H_{01}$ and $H_{02}$ as functions of $N$, together with the asymptotic solutions $(38)_{2}$ in the limit $N \rightarrow 0^{+}$and $(39)_{2}$ in the limit $N \rightarrow \infty$, represented by dashed curves. The results for $H_{01}$ are difficult to distinguish at this scale, and so the inset shows an enlargement of the $H_{01}$ curve.

Fig. .8. Plots of $\eta_{01}$ and $\eta_{02}$ as functions of $N$, together with the asymptotic solutions (38) 3 in the limit $N \rightarrow 0^{+}$and $(39)_{3}$ in the limit $N \rightarrow \infty$, represented by dashed curves.

Fig. .9. Asymptotic solution in the limit $N \rightarrow \infty$ for the case $H_{0}=H_{01}$ : comparison between $\bar{H}_{1}$ (represented by a dashed curve) and $N\left(H-\bar{H}_{0}\right)$ (represented by a full curve) for (a) $N=5$ and (b) $N=20$.

Fig. .10. As in Fig. .9, but for the case $H_{0}=H_{02}$.

Fig. .11. Three-dimensional plots of the free surfaces $h$ of the sessile rivulets for the cases (a) $H_{0}=H_{01}$ and (b) $H_{0}=H_{02}$ with $N=5$, at times $t=-1,-10$ and -100 . The insets show the cross-sectional profiles.

Fig. .12. Sketch of the asymptotic solution (38) for $H$ to $O(N)$ in the limit $N \rightarrow 0^{+}$for $H_{0}=H_{02}$.

Fig. .13. Profiles $H$ for the case $H_{0}=H_{02}$ obtained numerically (represented by a full curve) and from the asymptotic solution to $O(N)$ in the limit $N \rightarrow 0^{+}$given by (38) (represented by a dashed curve) for (a) $N=1 / 2$ and (b) $N=1 / 20$. 


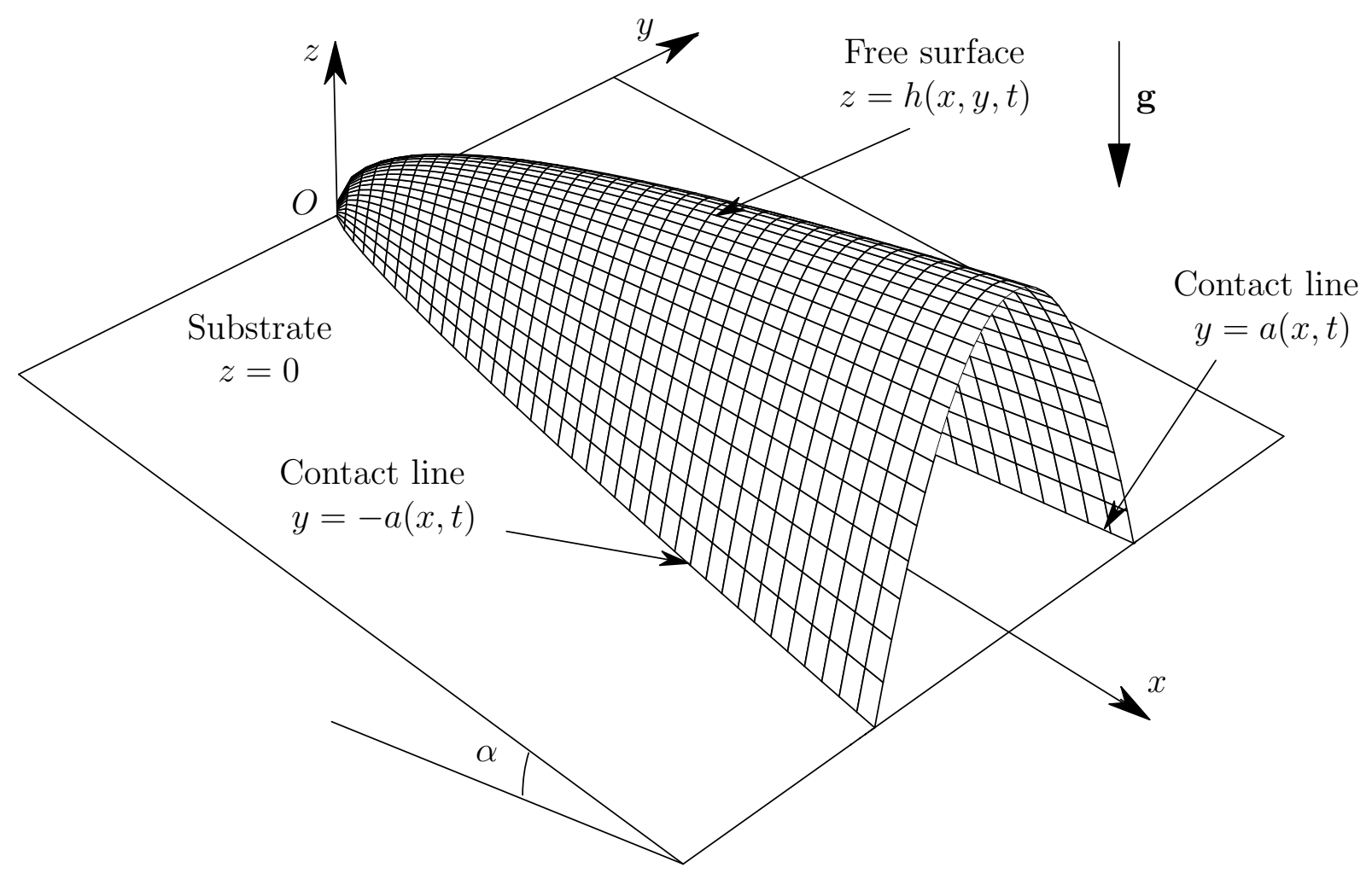

Figure .1: Sketch of the geometry of the problem. 


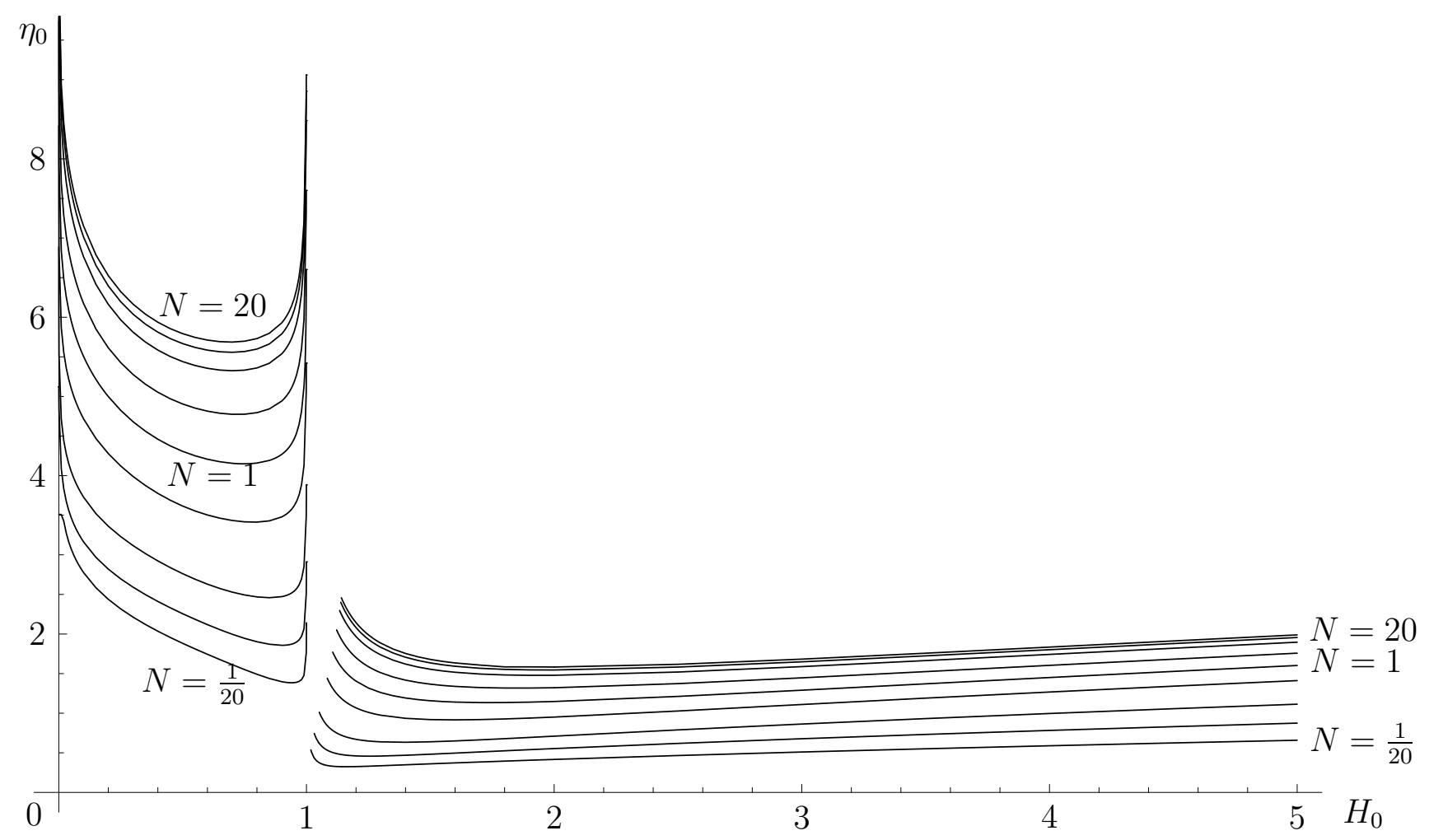

Figure .2: Plot of $\eta_{0}$ as a function of $H_{0}$ for $N=1 / 20,1 / 10,1 / 5,1 / 2,1,2,5,10$ and 20 . 


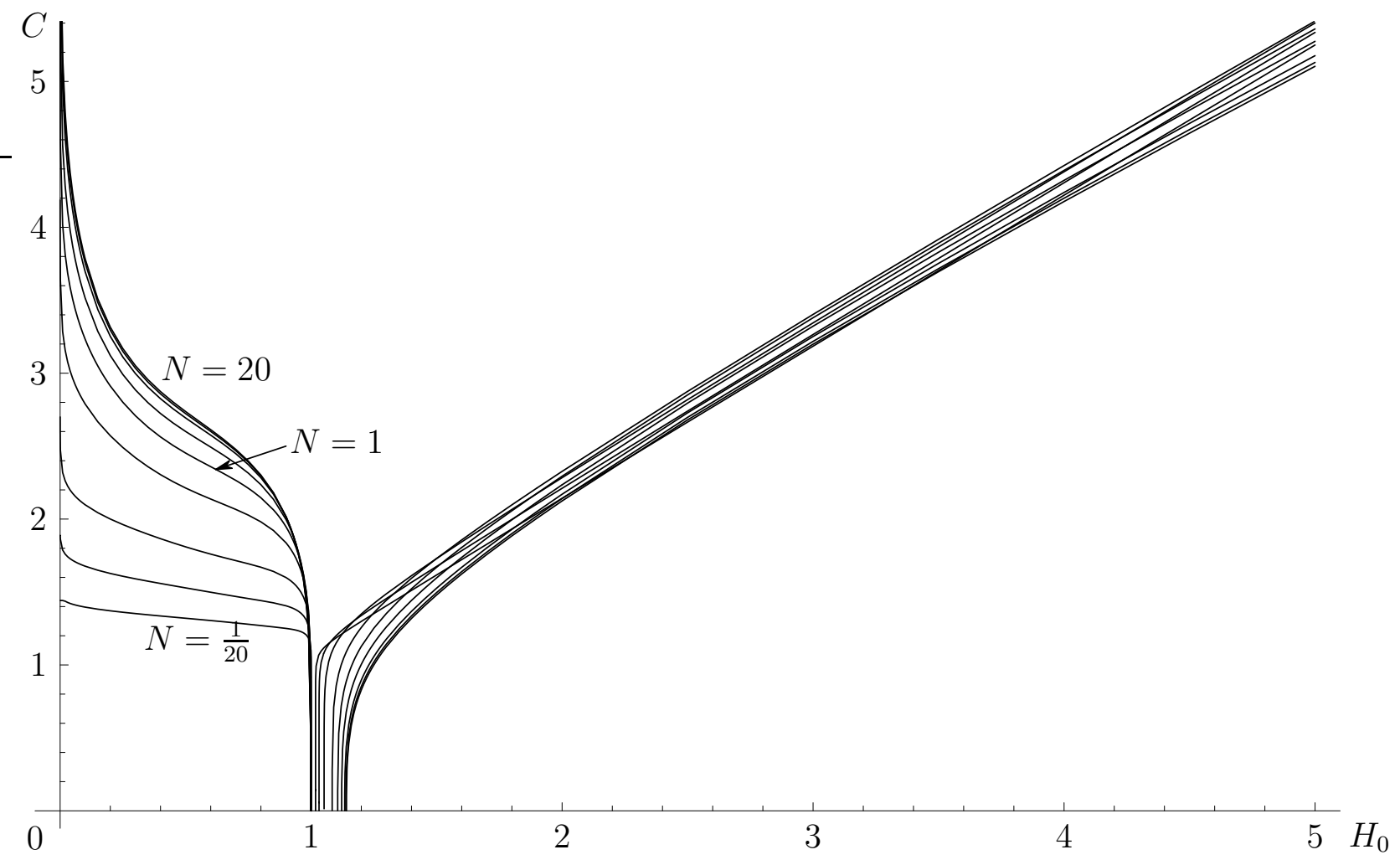

Figure .3: Plot of $C$ in (37) as a function of $H_{0}$ for $N=1 / 20,1 / 10,1 / 5,1 / 2,1,2,5,10$ and 20 . 


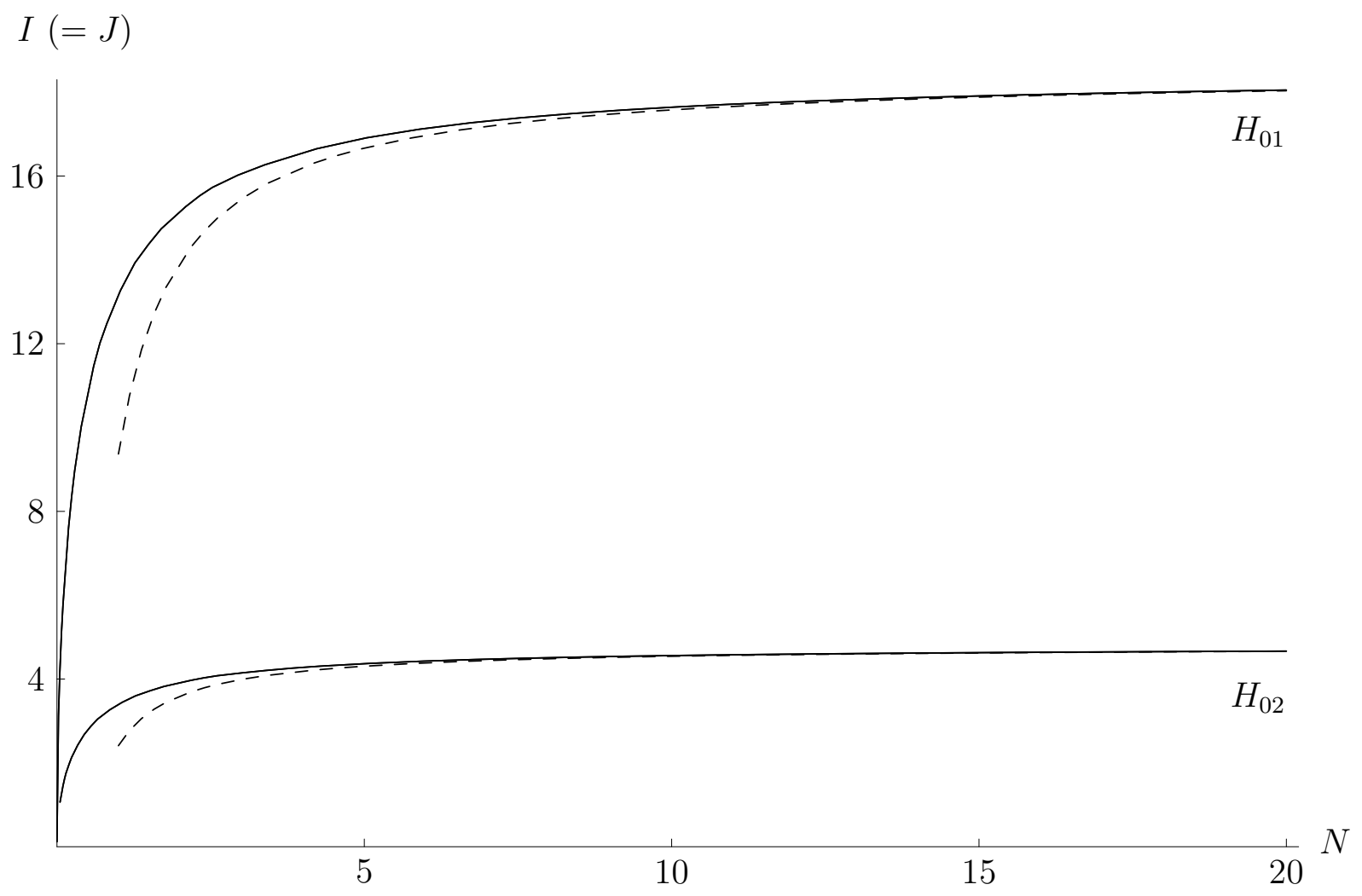

Figure .4: Plot of $I(=J)$ given by (24) as a function of $N$ for $H_{0}=H_{01}$ and $H_{0}=H_{02}$, together with the asymptotic solutions (38) in the limit $N \rightarrow 0^{+}$and (46) in the limit $N \rightarrow \infty$, represented by dashed curves. 


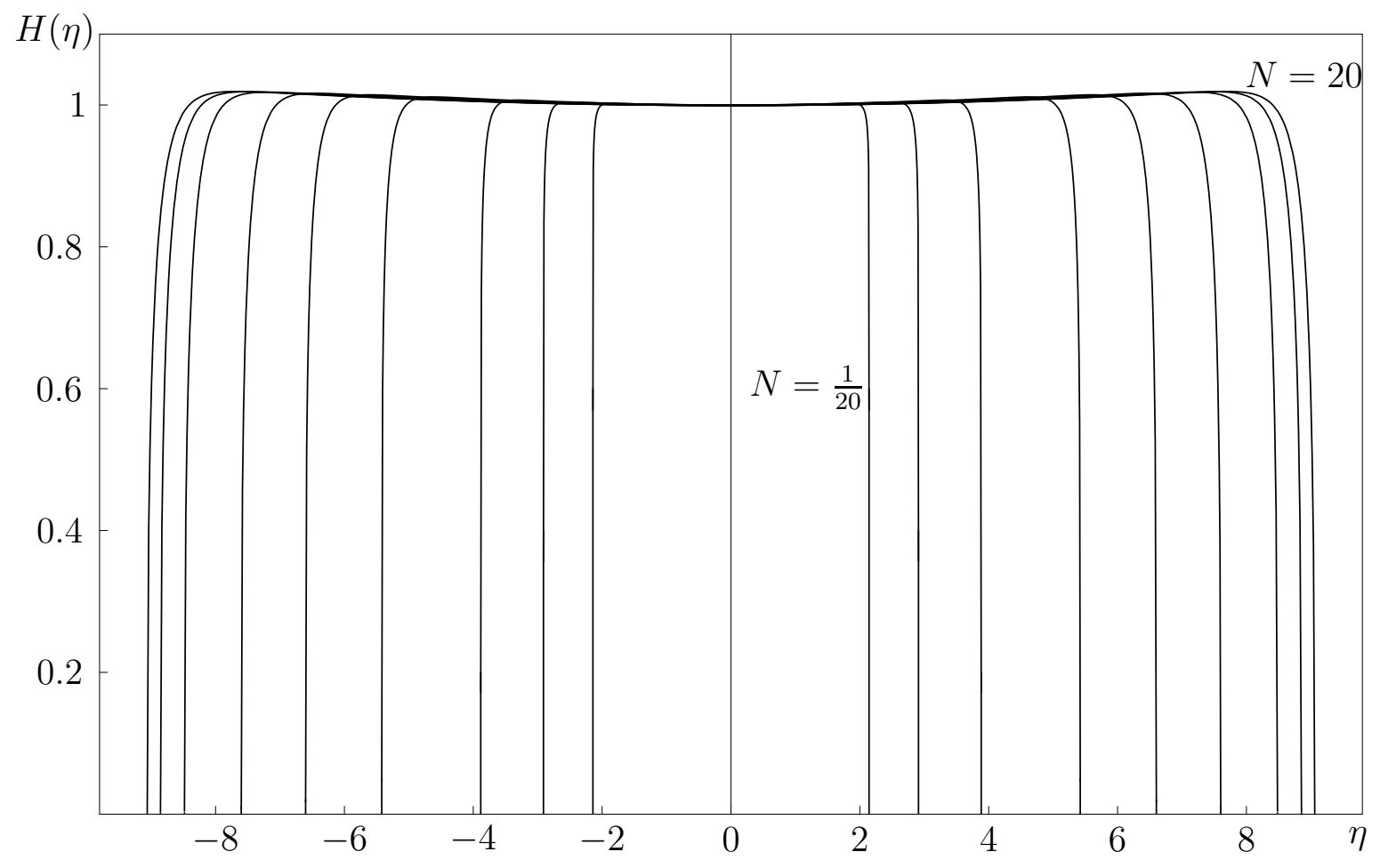

Figure .5: Numerically calculated profiles $H=H(\eta)$ for the case $H_{0}=H_{01}$, for $N=1 / 20,1 / 10,1 / 5,1 / 2,1,2$, 5,10 and 20 . 


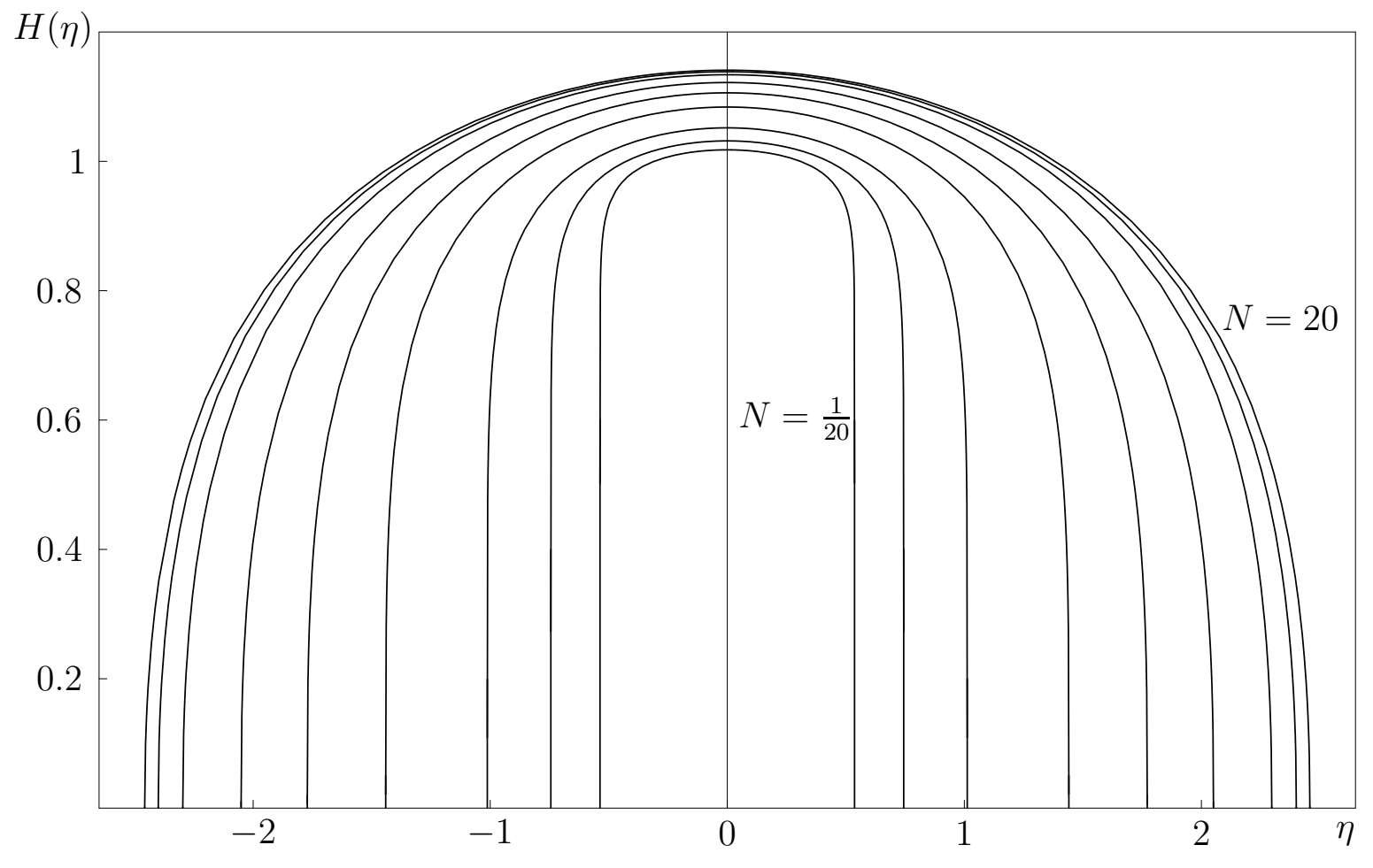

Figure .6: As in Fig. .5, but for the case $H_{0}=H_{02}$. 


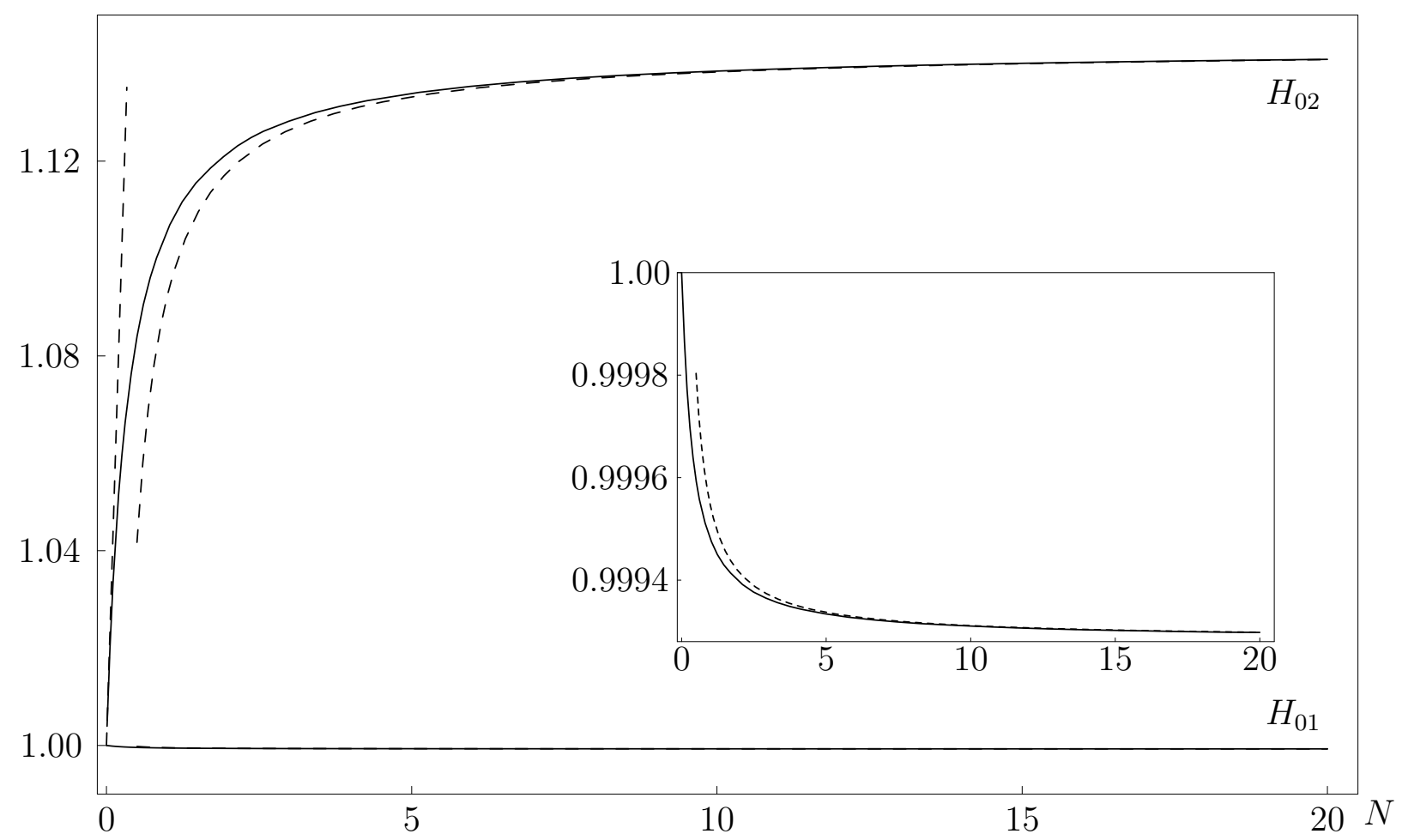

Figure .7: Plots of $H_{01}$ and $H_{02}$ as functions of $N$, together with the asymptotic solutions $(38)_{2}$ in the limit $N \rightarrow 0^{+}$and $(39)_{2}$ in the limit $N \rightarrow \infty$, represented by dashed curves. The results for $H_{01}$ are difficult to distinguish at this scale, and so the inset shows an enlargement of the $H_{01}$ curve. 


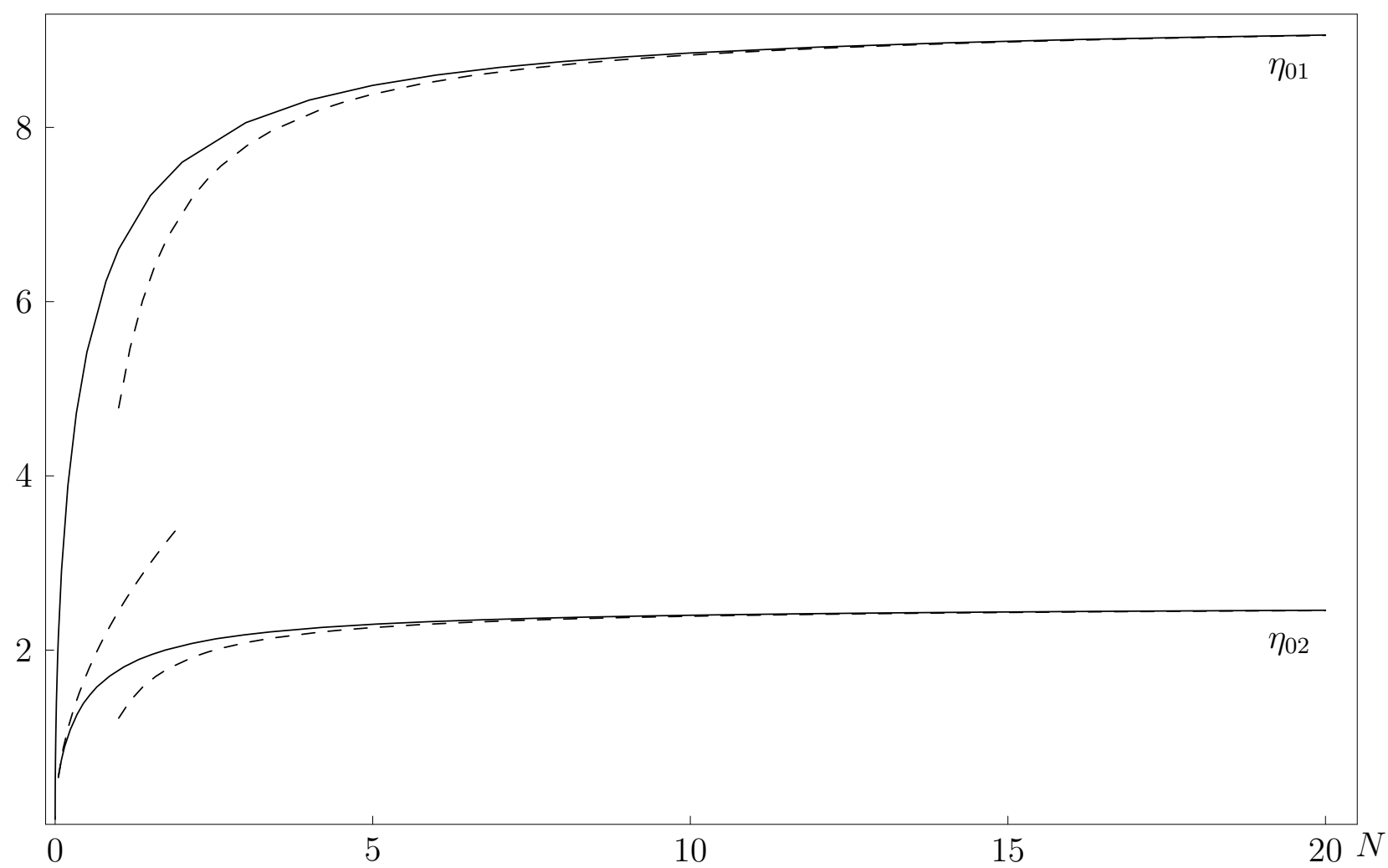

Figure .8: Plots of $\eta_{01}$ and $\eta_{02}$ as functions of $N$, together with the asymptotic solutions (38) 3 in the limit $N \rightarrow 0^{+}$and $(39)_{3}$ in the limit $N \rightarrow \infty$, represented by dashed curves. 
(a)

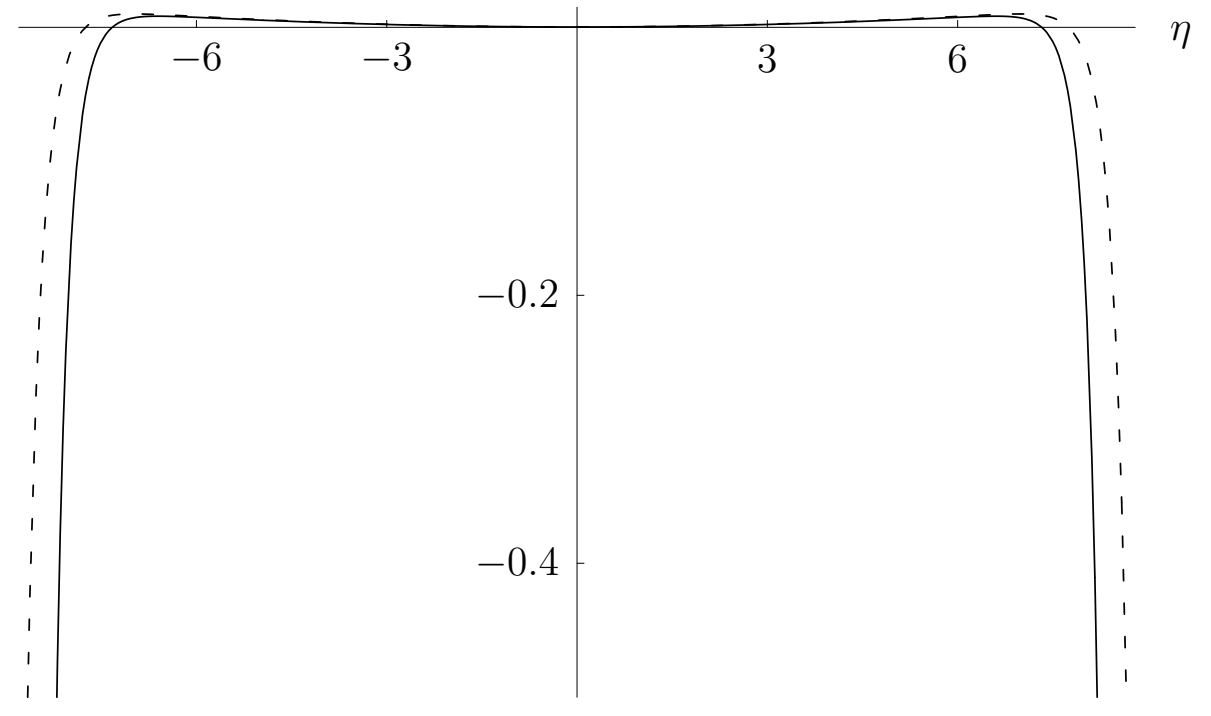

(b)

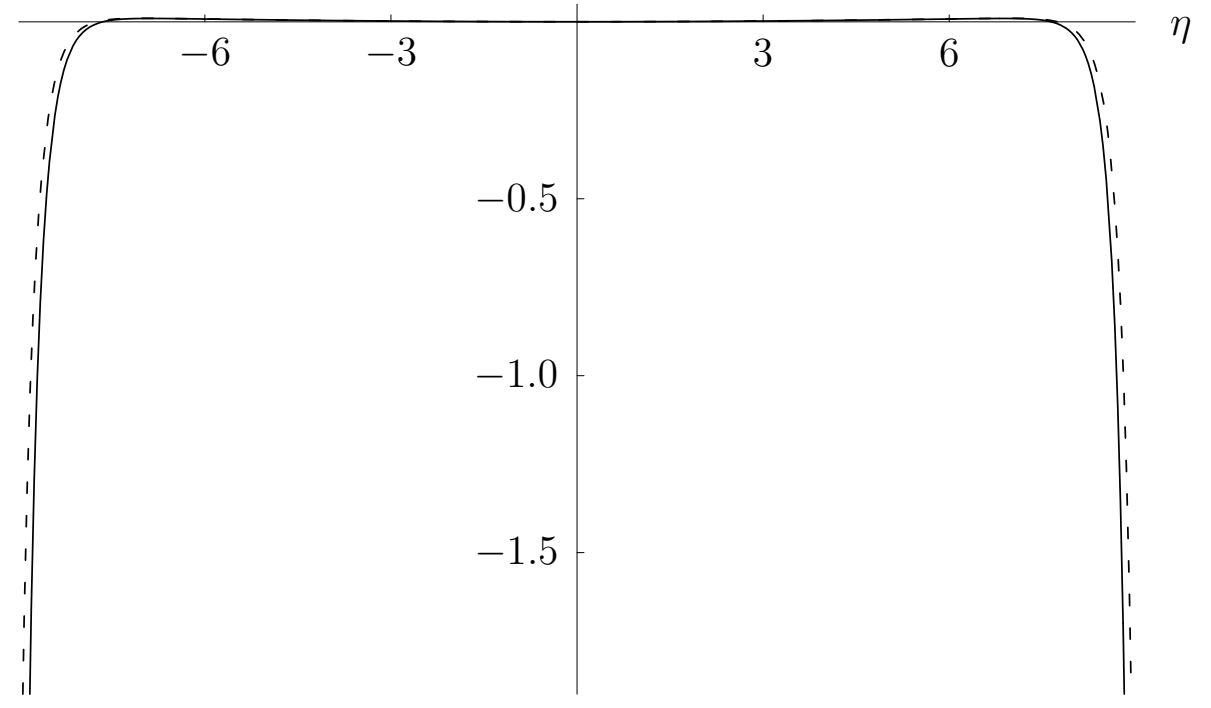

Figure .9: Asymptotic solution in the limit $N \rightarrow \infty$ for the case $H_{0}=H_{01}$ : comparison between $\bar{H}_{1}$ (represented by a dashed curve) and $N\left(H-\bar{H}_{0}\right)$ (represented by a full curve) for (a) $N=5$ and (b) $N=20$. 
(a)

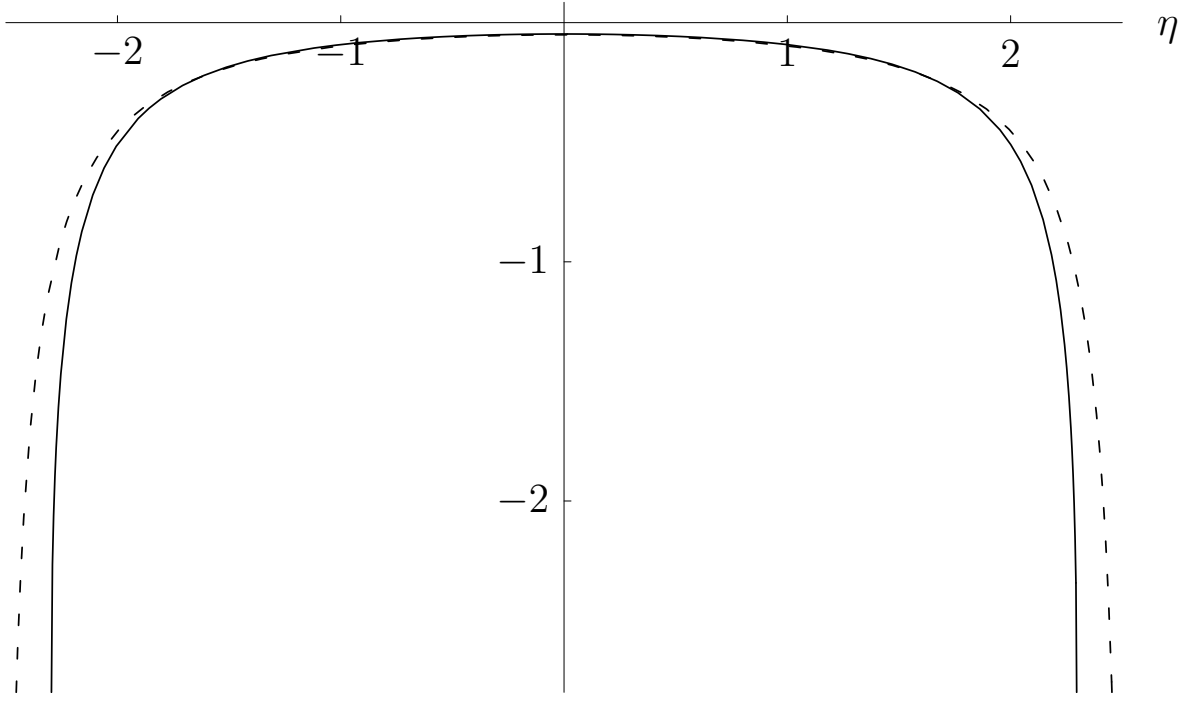

(b)

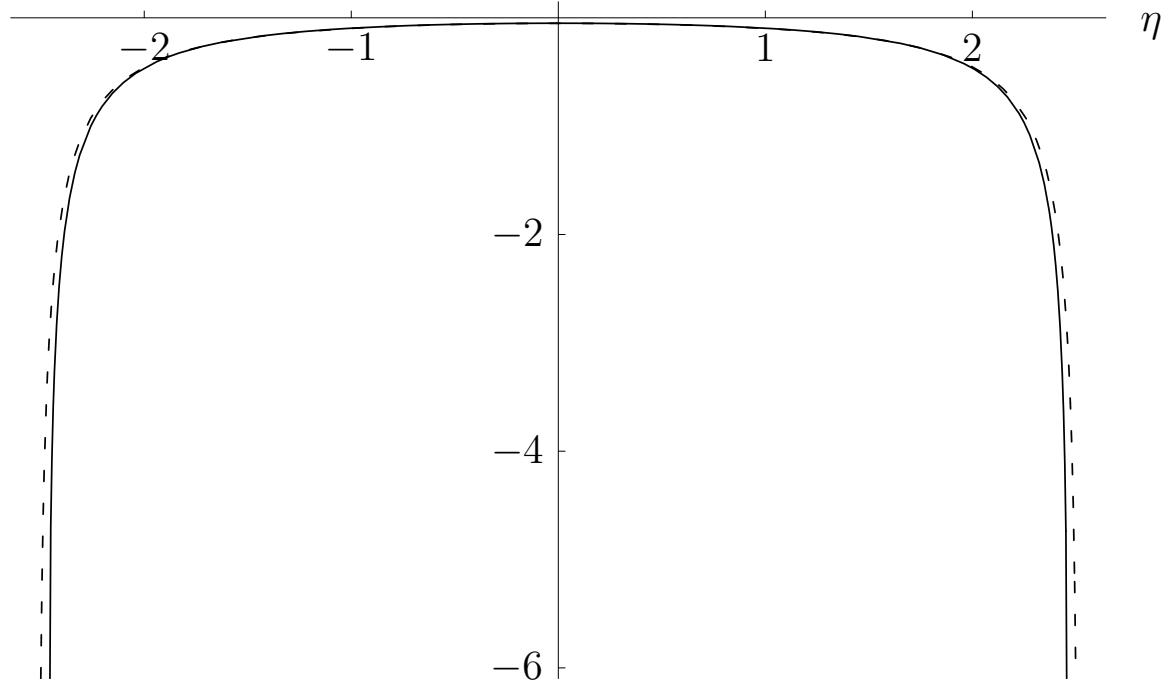

Figure .10: As in Fig. .9, but for the case $H_{0}=H_{02}$. 

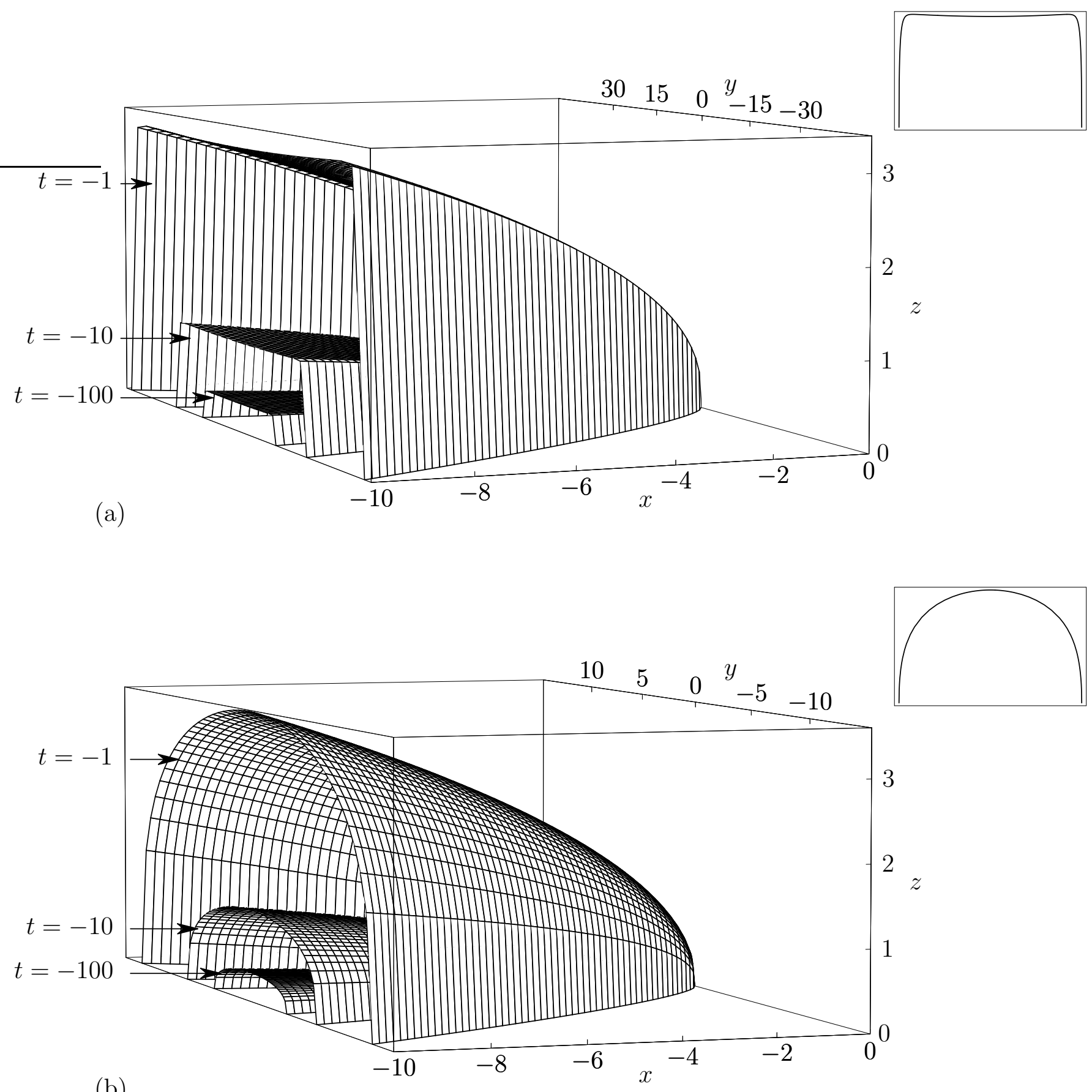

Figure .11: Three-dimensional plots of the free surfaces $h$ of the sessile rivulets for the cases (a) $H_{0}=H_{01}$ and (b) $H_{0}=H_{02}$ with $N=5$, at times $t=-100,-10$ and -1 . The insets show the cross-sectional profiles. 


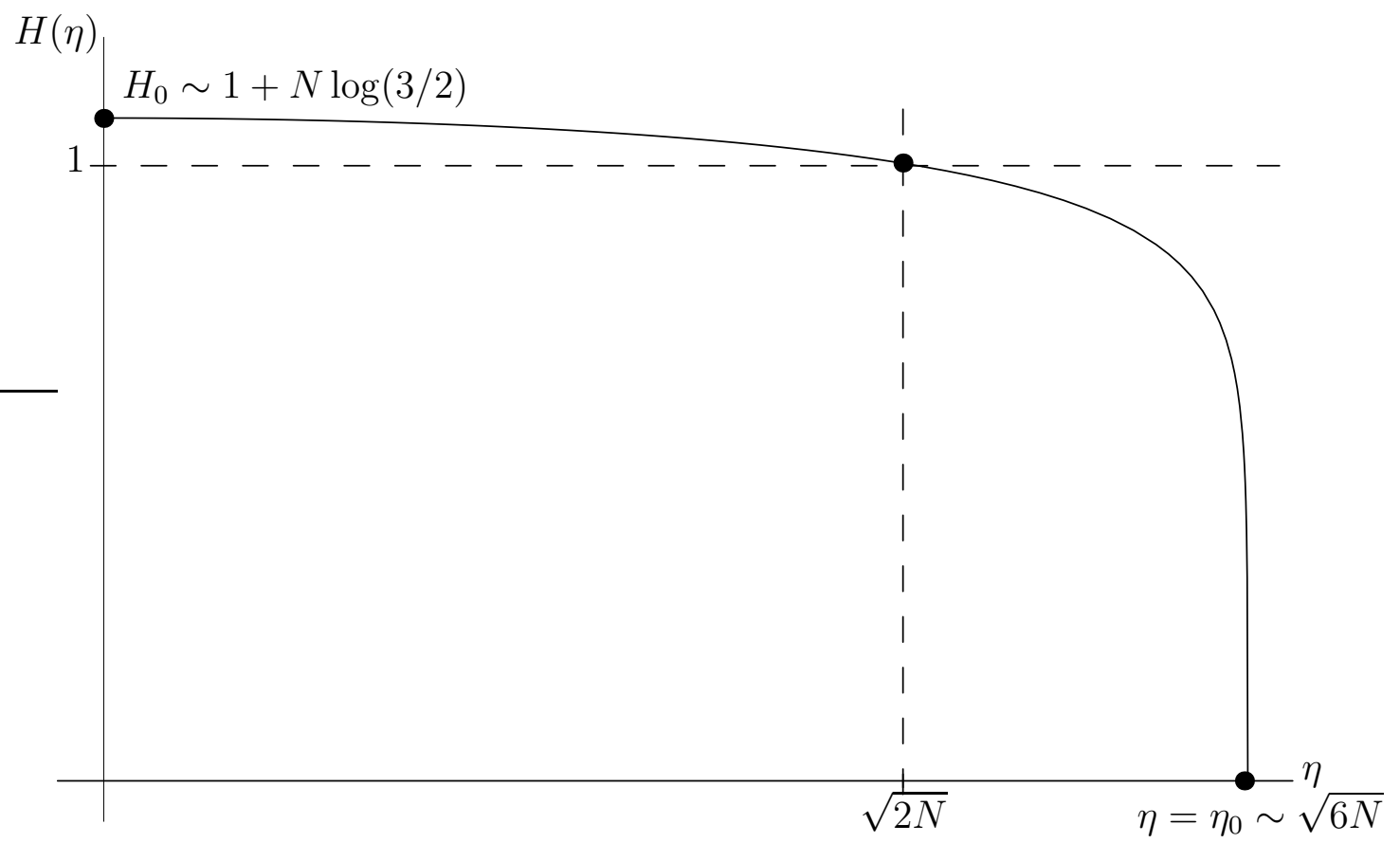

Figure .12: Sketch of the asymptotic solution (38) for $H$ to $O(N)$ in the limit $N \rightarrow 0^{+}$for $H_{0}=H_{02}$. 
(a)
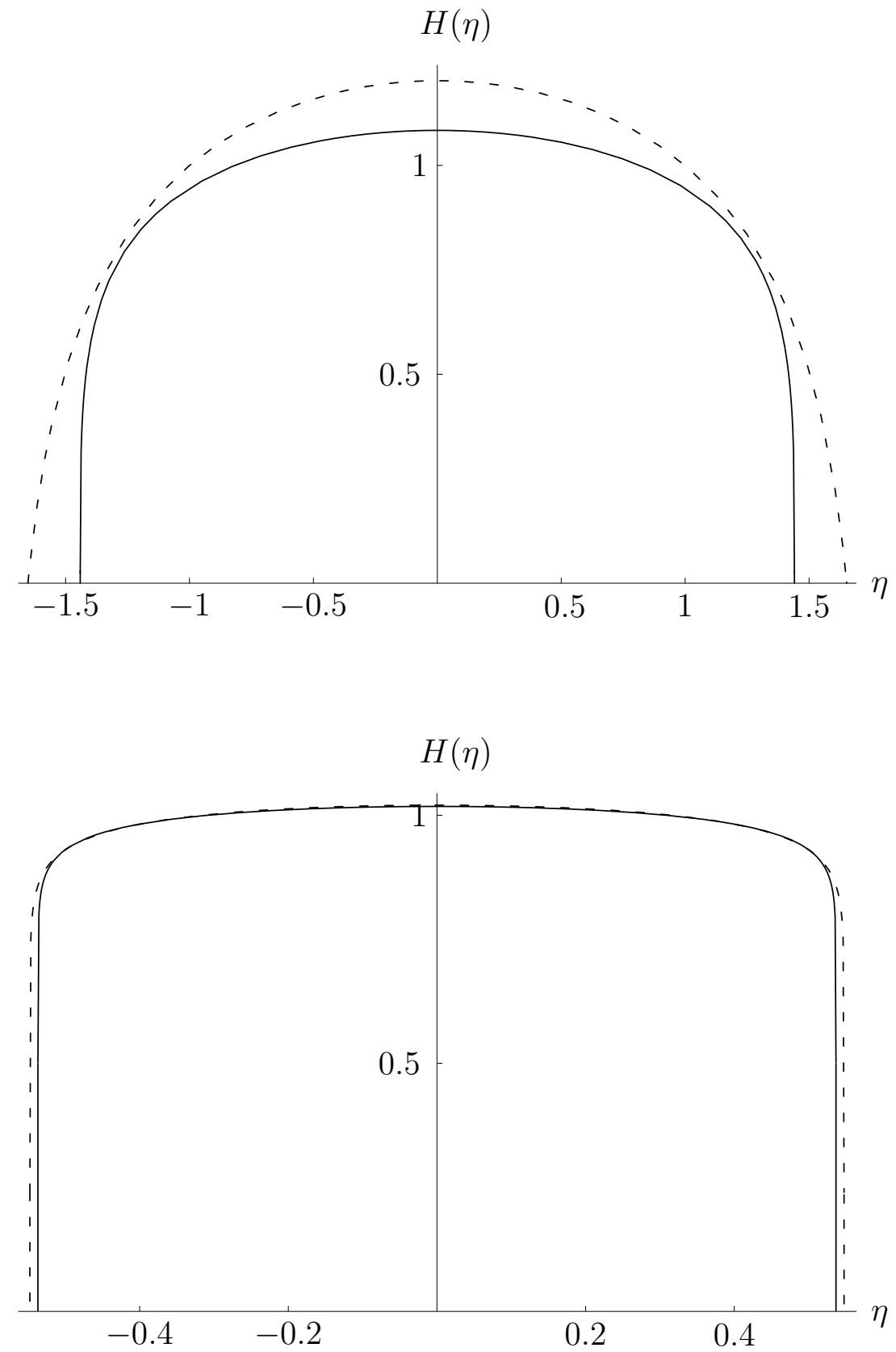

(b)

Figure .13: Profiles $H$ for the case $H_{0}=H_{02}$ obtained numerically (represented by a full curve) and from the asymptotic solution to $O(N)$ in the limit $N \rightarrow 0^{+}$given by (38) (represented by a dashed curve) for (a) $N=1 / 2$ and (b) $N=1 / 20$. 